\title{
Successful pregnancy in a patient with Swyer syndrome, or pure $46, X Y$ gonadal dysgenesis
}

\author{
A Chrysostomou, ${ }^{1}$ MD, FCOG (SA); $\mathbf{M}$ Tsuari, ${ }^{2}$ MMed (Obstetrics Gynaecology) \\ ${ }^{1}$ Department of Obstetrics and Gynaecology, Charlotte Maxeke Johannesburg Academic Hospital and Faculty of Health Sciences, University of the \\ Witwatersrand, Johannesburg, South Africa \\ ${ }^{2}$ Netcare Linkwood Hospital, Johannesburg, South Africa
}

Corresponding author: A Chrysostomou (Andreas.chrysostomou@wits.ac.za)

\begin{abstract}
Swyer syndrome, or pure 46,XY gonadal dysgenesis, is a rare disorder of sex development, characterised by the failure of sex gland development (ovaries or testes) in a phenotypic female patient. A 24-year-old woman with this syndrome presented at a tertiary academic hospital in South Africa, complaining of primary amenorrhoea and infertility. After gonadectomy and fertility treatment, a rare successful pregnancy outcome was achieved. A patient with Swyer syndrome, in a specialist fertility programme, can maintain a normal pregnancy and delivery.
\end{abstract}

S Afr J Obs Gynae 2019;25(1):9-11. DOI:10.7196/SAJOG.2019.v25i1.1448

The condition known as Swyer syndrome or pure 46,XY gonadal dysgenesis is a rare disorder; the exact incidence is unknown. It has been estimated to occur in 1 in 80000 births. Despite having an XY chromosomal makeup, girls or young women with Swyer syndrome are phenotypically female and have functional female genitalia and associated structures (uterus, fallopian tubes, cervix, vagina), and bilateral rudimentary streak gonads. As such, those with Swyer syndrome are typically raised as female and have a female gender identity, as well as a normal sexual life. Gonadectomy is recommended at the time of diagnosis, owing to the risk of malignant transformation in the gonadal streaks. ${ }^{[2,3]}$ A small number of successful pregnancies achieved with oocyte donation have been reported in patients with Swyer syndrome. ${ }^{[4-13]}$ We add to that record here by reporting a successful pregnancy in a patient with Swyer syndrome after the transfer of an embryo derived from donor eggs. Moreover, we believe that this is the first recorded such case in both South Africa and the African continent.

\section{Case report}

A 24-year-old nulliparous woman consulted for primary amenorrhoea at the gynaecological outpatient department of Charlotte Maxeke Johannesburg Academic Hospital, South Africa. The initial referral diagnosis was androgen insensitivity syndrome (formerly testicular feminisation). On physical examination, the patient was phenotypically female: height, $178 \mathrm{~cm}$; weight, $72 \mathrm{~kg}$; with normal secondary sexual characteristics (pubic and axillary hair was present, with breast development Tanner stage IV). Normal external genitalia with normal clitoris were observed. On speculum examination, vagina length was normal, the cervix appeared normal, and the Pap smear was reported to be normal.

On bimanual examination, the uterus was found to be of small size, and no adnexal masses were palpable. Investigations revealed the presence of elevated gonadotropins (follicle-stimulating hormone, luteinising hormone) and low levels of oestrogen.
Testosterone was absent. A karyotype study revealed that the patient had 46,XY chromosome, which suggested the diagnosis of Swyer syndrome. A vaginal ultrasound scan confirmed the clinical findings of a normal shape uterus but hypoplastic, measuring $4.1 \times 2 \mathrm{~cm}$; the ovaries could not be visualised.

The patient underwent diagnostic and operative laparoscopy, where rudimentary streak ovaries were evident. Laparoscopic bilateral gonadectomy and salpingectomy were performed, and the histology confirmed the features of gonads replaced with fibrous tissue (streak ovaries). There was no neoplasia. Postoperatively the patient was started on combined oestrogen/progestin treatment in the form of combined oral contraception (COC), resuming normal menstrual cycles with good evolution. Two years after her initial presentation and treatment (including gonadectomy), the patient and her husband expressed an interest in pursuing pregnancy using an egg donor. The patient was introduced to the oocyte donation programme, and fell pregnant. The pregnancy was closely monitored. A healthy baby with an Apgar score of 9 was delivered by caesarean section at 38 weeks' gestation.

\section{Discussion}

Since the first description of Swyer syndrome in 1955, a number of cases have been reported. Pure 46,XY gonadal dysgenesis, also called Swyer syndrome, is characterised by 46,XY karyotype in a phenotypically female patient. Despite having the XY chromosome, girls or young women with Swyer syndrome appear quite normally female and have functional female genitalia and structures, including a vagina, uterus, cervix and fallopian tubes. According to the new classification of disorders of sex development (DSD), Swyer syndrome falls under the XY umbrella of DSD. The new (2008) proposed nomenclature is 46,XY complete gonadal dysgenesis, which encompasses any disorder in which chromosomal, gonadal or anatomic sex development is abnormal. ${ }^{[14]}$ 
Pathogenesis has been described during embryogenesis, where the early stages of genital development are similar in the male and female. Normal development in the female involves the growth of Müllerian elements and the atrophy of Wolffian ones. Moreover, the gonad itself in its early stage of genital development remains for a short period of time in a neutral stage (indifferent gonads) during which it is capable of developing into a testis or an ovary. The first step of sexual differentiation of a normal XY fetus is the development of testes, during the second month of gestation. This requires the action of several genes, the most important being SRY, the sex-determining region of the $\mathrm{Y}$ chromosome. Mutations of SRY account for 10 - 15\% of cases of complete gonadal dysgenesis (Swyer syndrome).$^{[14]}$ In Swyer syndrome (46,XY pure gonadal dysgenesis), the indifferent gonads fail to differentiate into testes in an $\mathrm{XY}$ (genetically male) fetus. In the absence of testes, no testosterone or anti-Müllerian hormone (AMH) is produced. Without testosterone, the genitalia fail to virilise, resulting in normal female genitalia. The Wolffian ducts fail to develop, so no internal male organs are present. Without $\mathrm{AMH}$, the Müllerian ducts develop into normal internal female organs (uterus, fallopian tubes, cervix and vagina).

As in the present case, the individual develops a normal female identity, although complicated by primary amenorrhoea and the possession of non-functional streak gonads instead of ovaries or testes. Before puberty (even in normal females), the ovaries play little or no role in bodily changes. Therefore the problem manifests at puberty, owing to the inability of the streak gonads to produce sex hormones (both oestrogens and androgens). Most secondary sexual characteristics do not develop, and there is a lack of menses in the majority of phenotypically female patients with pure gonadal dysgenesis.

In this case, the secondary sexual characteristics did develop, as pubic and axillary hair were present. This is attributable to the limited amount of androgen produced by adrenal glands, which are not affected by this syndrome. The breasts were developed, but not fully, as the main source of oestrogens was the peripheral aromatisation of androgens. This patient's main complaint was primary amenorrhoea. Having been sexually active for 3 years, primary infertility was also a concern expressed at the first tertiary centre presentation.

Age of diagnosis is important in the management of Swyer syndrome, as the gonads of XY pure gonadal dysgenesis have a high risk of gonadal malignancy, such as a gonadoblastoma and germ cell tumour. ${ }^{[2,3]}$ The postoperative evolution was uneventful, and the patient was started on COC on resuming menses. It is important to note that slow introduction of oestrogen may improve breast development.

In the case of this patient, diagnosis had been delayed, and was only confirmed at the age of 24 years. This case of a delayed diagnosis of Swyer syndrome 6 years after the initial consultation with the general practitioner was reported previously by the first author. ${ }^{[15]}$ A study by Michala et al. ${ }^{[4]}$ has shown that many women experience a delay in reaching an accurate diagnosis, often several years after the first presentation to their general practitioners. Delay in diagnosis is often due to a normal phenotypic appearance.

There is a need to increase scientific knowledge and awareness of the disorder among health professionals. Early diagnosis is important, not only because of the risk of gonadal malignancy, but also owing to the vital role that early institution of hormonal therapy plays in the induction of puberty. Hormone therapy may also be required to improve bone mineral density and to prevent osteoporosis.

Upon diagnosis, the patient and her husband were counselled with regard to her condition. Their understanding was ensured and she was referred to a genetic counselling centre, where they received guidance and support regarding the diagnosis, complications and outcomes. As seen in our case, patients with Swyer syndrome can achieve pregnancy through a donated oocyte programme and hormonal support during the early antenatal period. The presence of an XY genotype does not affect normal uterine and endometrial response, and the potential for patients to maintain a normal pregnancy and delivery confirms the physiological ability of the uterus to accommodate and maintain a successful pregnancy. As in our patient's case, almost all reported pregnancies in patients with Swyer syndrome were delivered by caesarean section. This decision was based not on clear obstetric indications, but patient request. Others have considered that caesarean delivery may be more appropriate owing to a possible androgenic shape of the pelvis (though it is not obvious that this is correct), possible unstable lie of the baby and the hypoplastic uterus. Fewer than 13 live births have been reported in patients with Swyer syndrome. ${ }^{[5-13]}$

Patients with 46,XY pure gonadal dysgenesis do not appear to be at increased risk for the adverse pregnancy outcomes seen in patients with 45,X gonadal dysgenesis (Turner syndrome) or $46, \mathrm{Y}$ mosaicism, which are mainly cardiovascular anomalies (e.g. aortic dilatation, coarctation or bicuspid aortic valve). ${ }^{[16]}$ Height is particularly helpful in distinguishing patients with $46, \mathrm{XY}$ pure gonadal dysgenesis from those with $45 \mathrm{X} / 46, \mathrm{XY}$ mosaicism. ${ }^{[17]}$

\section{Conclusion}

This case highlights the role of laparoscopy in not only the diagnosis but also the management of Swyer syndrome. The primary care physician needs to be aware of this condition, as early referral to tertiary centres is necessary for appropriate management.

A patient with 46,XY pure gonadal dysgenesis, in a specialist fertility programme, can establish and then maintain a normal pregnancy.

Acknowledgements. None.

Author contributions. Equal contributions.

Funding. None.

Conflicts of interest. None.

1. Coutin AS, Hamy A, Fondevilla M, Savingy B, Paineaou J, Vissez J. Pure 46,XY gonadal dysgenesis. J Gynecol Obst Biol Reprod 1996,25(8):792-796.

2. Nadereh B, Mojgan KZ. Dysgerminoma in three patients with Swyer syndrome. World J Surg Oncol 2007;5:71. https://doi.org/10.1186/1477-7819-5-71

3. Zielinska R, Zajaczek S, Rzepka-Górska I. Tumours of genetic gonads in Swyer syndrome. J Pediatr Surg 2007;42(10):1721-1724. https://doi.org/10.1016/j.jpedsurg.2007.05.029

4. Michala L, Goswami D, Creighton SM, et al. Swyer syndrome: Presentation and outcomes. BJOG 2008;115(6):735-741. https://doi.org/10.1111/j.1471-0528.2008.01703.x

5. Beth JP, Marc AF. A case report of successful pregnancy in a patient with pure $46, \mathrm{XY}$ gonadal dysgenesis. Fertil Steril 2008;90:5

6. Tulic I, Tulic L, Misic J. Pregnancy in patient with Swyer syndrome. Fertil Steril 2011;95(5):1789.

7. Ko PC, Peng HH, Soong Y, Chang K. Triplet pregnancy complicated with one hydatidiform mole and preeclampsia in a 46,XY female with gonadal dysgenesis. Taiwan J Obstet Gynecol 2007;46(3):276-280. https://doi.org/10.1016/S1028-4559(08)60034-0

8. Dirnfeld M, Bider D, Abramovici H, Calderon I, Blumenfeld Z. Subsequent successful pregnancy and delivery after intracytoplasmic sperm injection in patient with XY gonadal dysgenesisms. Eu

9. Sauer MV, Lobo RA, Paulson RJ. Successful twin pregnancy after embryo donation to a patient with XY gonadal dysgenesis. Am J Obstet Gynecol 1989:161:380-381

10. Chen MJ, Yang JH, Mao TL, Ho HN, Yang YS. Successful pregnancy in a gonadectomised woman with 46,XY gonadal dysgenesis and gonadoblastoma. Fertil Steril 2005;84(1):217. https://doi. org/10.1016/j.fertnstert.2004.11.087 


\section{CASE REPORT}

11. Kan AK, Abdalla HI, Oskarsson T. Two successful pregnancies in a 46,XY patient. Hum Reprod 1997;12:1434-1435

12. Sauer MV, Lobo RA, Paulson RJ. Successful twin pregnancy after embryo donation to a patient with XY gonadal dysgenesis. Am J Obstet Gynecol 1989;161(1):380-381. https://doi.org/10.1016/0002 9378(89)90525-5

13. Selvaraj K, Ganesh V, Selvaraj P. Successful pregnancy in a patient with $46, \mathrm{XY}$ karyotype. Fertil Steril 2002;78:419-420.

14. Hughes LA. Disorders of sex development: A new definition and classification. Best practice and research. Clin Endocrinol Metabol 2008;22(1):119-134. https://doi.org/10.1016/j.beem.2007.11.001

15. Chrysostomou A. Primary amenorrhoea: Swyer syndrome in a woman with pure $46, \mathrm{XY}$ gonada dysgenesis and late presentation. S Afr J Obstet Gynae 2015;21(1);16-17. https://doi.org/10.7196/ SAJOG.2018.v24i2.127

16. Practice Committee of the American Society for Reproductive Medicine. Increased materna cardiovascular mortality associated with pregnancy in women with Turner syndrome. Fertil Sterl 2006;86(5 Suppl 1):S127-S128.

17. McDonough PG. In syndromes of confused gonads, height can be important! Fertil Steril 2003;79(2):462-463. https://doi.org/10.1016/s0015-0282(02)04770-2

Accepted 29 April 2019. 